\begin{tabular}{|c|l|}
\hline Title & Electron and ion transport through multilayers of A u nanoclusters covered by self-assembled monolayers \\
\hline Author(s) & Song, Wenbo; Okamura, Masay uki; Kondo, Toshihiro; U osaki, Kohei \\
\hline Citation & $\begin{array}{l}\text { Journal of Electroanalytical Chemistry, 554-555, 385-393 } \\
\text { https:/doi.org/10.1016/50022-0728(03)00303-6 }\end{array}$ \\
\hline Issue Date & $2003-09-15$ \\
\hline Doc URL & http://hdl.handle.net/2115/20571 \\
\hline Type & article (author version) \\
\hline File Information & jec554-555.pdf \\
\hline
\end{tabular}

Instructions for use 


\title{
Electron and Ion Transport through Multilayers of Au Nanoclusters Covered by Self-Assembled Monolayers
}

\author{
Wenbo Song, Masayuki Okamura, Toshihiro Kondo, and Kohei Uosaki* \\ Physical Chemistry Laboratory, Division of Chemistry, Graduate School of Science, \\ Hokkaido University, Sapporo 060-0810, Japan
}

\begin{abstract}
Two types of gold nanoclusters (GNCs), one covered by self-assembled monolayers (SAMs) of mercaptoundecanoic acid (MUA), hexanethiol $\left(\mathrm{C}_{6} \mathrm{SH}\right)$, and ferrocenylhexanethiol $\left(\mathrm{FcC}_{6} \mathrm{SH}\right), \mathrm{MHF}-\mathrm{GNC}$, and the other with MUA and $\mathrm{C}_{6} \mathrm{SH}, \mathrm{MH}-\mathrm{GNC}$, were used for the construction of ten GNC layers of two different sequences on an MUA modified $\mathrm{Au}(111)$ surface based on the carboxylate/polycation (poly(allylamine hydrochloride) : $\mathrm{PAH}$ )/carboxylate electrostatic interaction. MHF-GNC was placed either as the layer closest to the gold electrode, i.e., the first layer, or as the outermost layer with $\mathrm{MH}-\mathrm{GHC}$ in the other layers. A quasi-reversible redox peak with a constant charge corresponding to the redox of the ferrocene moiety of the MHF-GNC monolayer was observed at both electrodes, showing electrons and perchlorate ions could be transferred through the MH-GNC/PAH multilayers. Cross-sectional transmission electron microscopy revealed that the size of the immobilized GNC was almost same as that in solution and well-separated GNCs were dispersed rather uniformly within the polymeric matrix. The incorporation of perchlorate ion upon the oxidation of the ferrocene moiety of the MHF-GNC was demonstrated by electrochemical quartz microbalance measurement. Based on the above results, the charge transfer mechanism in the GNC multilayers was discussed.
\end{abstract}




\section{INTRODUCTION}

The formation of ordered mono- and multi- layers of both organic and inorganic materials on solid substrates with various functionalities has been a subject of intensive research in view of the possible applications in a wide variety of fields in nanotechnology and nanoscience.

One of the good candidates as a building block for the multilayer formation is metal and semiconductor nanoclusters because they have various interesting characteristics, which are different from those of the bulk materials. Since the report by Brust et al. [1], gold nanoclusters (GNCs) covered by self-assembled monolayers (SAMs) of alkanethiols have attracted many research groups [2-14] because SAM-covered GNCs have a high stability and those of a certain size with a narrow size distribution can be prepared relatively easily. GNC with special functionalities can be constructed by using thiol molecules with special functional groups. One can utilize the rich references on alkanethiol SAMs with various functionalities [15,16] including ours [17-20].

Several groups have already reported the formation and the properties of the GNC multilayers on solid surfaces [6,10-12a,12c,13,14,21]. For example, Murray et al. constructed the GNC mono- and multi- layers and reported the quantized charging and dynamics of electron transfer between the GNCs [10-12]. The electrostatic layer-by-layer (LBL) assembly method has been applied not only for the multilayer formation of oppositely charged polyelectrolytes [22], for which this method was originally developed, but also to construct nanoparticle multilayers [12c, 23, 24] by alternatively immersing the charged substrate surface into oppositely charged GNC and ionic polymeric solutions with a rinse after each deposition. Combining the versatility of polymeric materials with GNCs by LBL sequential formation of ordered nanostructures offers significant advantages such as usage of environmentally-friendly aqueous solution, low cost, high throughput. However, the studies of the electron transport within the multilayer of GNCs covered by thiol molecules containing 
electroactive groups are rather limited. Recently, we have constructed the multilayers of GNCs covered by mixed self-assembled monolayers with an electrochemical active group, ferrocene, based on this versatile technique, and investigated their electrochemical properties $[26,27]$. In these reports, we found that the electric charge due to the redox of a ferrocene $(\mathrm{Fc}) /$ ferricenium cation $\left(\mathrm{Fc}^{+}\right)$couple linearly increased with the number of layers up to a certain number of layers. Preliminary results of the electrochemical characteristics of two types of the GNC multilayers with the Fc attached GNC either only at the outermost layer or at the closest layer to a gold substrate were also reported in these works to clarify the charge transfer mechanism within the multilayers. Electron was proposed to be transferred between ferrocene and the gold electrode through the GNC cores.

The understanding of electron transfer mechanism at the multilayer modified electrode/electrolyte interface is very important not only for fundamental surface science and electrochemistry but also for the design and fabrication of advanced electronic, optical and sensing nanodevices. In this study, we have investigated the electron transport properties within the multilayers of two GNCs, one covered by self-assembled monolayers of mercaptoundecanoic acid (MUA), hexanethiol $\left(\mathrm{C}_{6} \mathrm{SH}\right)$, and ferrocenylhexanethiol $\left(\mathrm{FcC}_{6} \mathrm{SH}\right)$, MHF-GNC, and the other with MUA and $\mathrm{C}_{6} \mathrm{SH}$, MH-GNC, with various sequences constructed based on a carboxylate/cationic polymer (poly(allylamine hydrochloride): $\mathrm{PAH} /$ carboxylate electrostatic interaction on a $\mathrm{Au}(111)$ surface. It is shown that all the ferrocene groups within the multilayer are electrochemically active even when the Fc attached GNC existed either only at the outermost layer or only at the closest layer to a gold substrate with 1 - 9 layers of GNC without Fc groups. This means electron and perchlorate ion can be transferred through the GNC multilayers and electron transfer between the ferrocene moiety and the $\mathrm{Au}(111)$ electrode takes place through the GNC cores by hopping. Cross-sectional transmission electron microscopy has revealed that no aggregation of the GNC within the multilayers. The incorporation of perchlorate ion upon the oxidation of the ferrocene moiety 
of the MHF-GNC is demonstrated by electrochemical quartz microbalance (EQCM) measurement. Based on the above results, the charge transfer mechanism in the GNC multilayers is discussed.

\section{EXPERIMENTAL}

Hydrogen tetrachloroaurate $\left(\mathrm{HAuCl}_{4}, 99.99 \%\right)$, sodium tetrahydroborate $\left(\mathrm{NaBH}_{4}\right)$, ethanol (superpure grade), methanol (superpure grade), hexanethiol $\left(\mathrm{C}_{6} \mathrm{SH}\right.$, superpure grade), calcium hydride $\left(\mathrm{CaH}_{2}\right)$, dichloromethane (ultrapure grade), perchloric acid (ultrapure grade), and sulfuric acid (ultrapure grade) were purchased from Wako Pure Chemicals. Tetra- noctylammonium bromide (TOAB) was obtained from Tokyo Kasei Kogyo. Tetra-nbutylammonium perchlorate (TBAP, for polarography use) was obtained from Nacalai Tesque, and 11- mercaptoundecanoic acid (MUA, 97\%) and toluene (spectroscopy grade) were obtained from Dojindo Laboratory. All chemicals except for dichloromethane and TBAP were used as received. Dichloromethane was distilled twice with $\mathrm{CaH}_{2}$ to remove any residual water. TBAP was recrystallized from methanol more than twice. Ferrocenylhexanethiol $\left(\mathrm{FcC}_{6} \mathrm{SH}\right)$ was synthesized by a previously reported method [27]. Ultrapure water was obtained using a Milli-Q water purification system (Millipore) and ultrapure $\mathrm{N}_{2}(99.999 \%)$ was obtained from Air Water.

A gold single crystal was prepared from a gold wire $(99.999 \%, \phi=1 \mathrm{~mm}$, Tanaka Precious Metals) by Clavilier 's method [29]. It was cut to expose the (111) face, mechanically polished, and then annealed at $800{ }^{\circ} \mathrm{C}$ for $8 \mathrm{~h}$ in an electric furnace (Denken, KDF S-70) under an ultrapure $\mathrm{N}_{2}$ atmosphere. Gold substrates used for the EQCM measurements were prepared by the vacuum evaporation of $10 \mathrm{~nm}$ thick titanium followed by $100 \mathrm{~nm}$ thick gold onto a $5 \mathrm{MHz}$ quartz crystal plate (double-side polished) kept at $300{ }^{\circ} \mathrm{C}$ with an evaporation rate of less than $0.01 \mathrm{~nm} \mathrm{~s}^{-1}$. This procedure is known to provide a highly ordered $\mathrm{Au}(111)$ phase with wide terraces [30]. For the XPS measurements, a gold disk 
$(99.99 \%, \phi=8 \mathrm{~mm}$, thickness $=3 \mathrm{~mm}$, Tanaka Precious Metals) was used as the substrate after mechanical polishing and electrochemical treatment by cycling the potential between 0.2 and $+1.5 \mathrm{~V}$ vs. $\mathrm{Ag} / \mathrm{AgCl}$ in $0.1 \mathrm{M} \mathrm{H}_{2} \mathrm{SO}_{4}$. All the gold substrates were annealed by a hydrogen flame and cooled in air just before dipping in the thiol solution.

Two types of GNCs covered with mixed alkanethiol SAMs, one covered by the SAMs of hexanethiol, ferrocenylhexanethiol, and 11-mercaptoundecanoic acid, MHF-GNC, and the other covered by $\mathrm{C}_{6} \mathrm{SH}$ and MUA, MH-GNC, were synthesized as follows: First, the $\mathrm{C}_{6} \mathrm{SH}$ SAM protected gold nanocluster, H-GNC, was prepared by the method reported by Brust et al. [1]. An aqueous solution of $\mathrm{HAuCl}_{4}(100 \mathrm{ml}, 20 \mathrm{mM})$ was mixed with a toluene solution of TOAB $(300 \mathrm{ml}, 30 \mathrm{mM})$. The two-phase mixture was vigorously stirred until all the $\mathrm{HAuCl}_{4}$ was transferred to the organic layer, i.e., the color of the organic phase changed to dark orange, and $\mathrm{C}_{6} \mathrm{SH}(0.68 \mathrm{~g}, 5.8 \mathrm{mmol})$ was then added to the organic phase. After stirring the mixture for $10 \mathrm{~min}$, a freshly prepared aqueous solution of $\mathrm{NaBH}_{4}(100 \mathrm{ml}, 260 \mathrm{mM})$ was slowly added with vigorous stirring. After further stirring the mixture vigorously for $3 \mathrm{~h}$, the organic phase was extracted by dichloromethane. The volume of the organic residue was reduced to $10 \mathrm{ml}$ in a rotary evaporator, and then $400 \mathrm{ml}$ of ethanol was added to remove the excess $\mathrm{C}_{6} \mathrm{SH}$. After the mixture was kept overnight at $-18{ }^{\circ} \mathrm{C}$, the dark brown precipitate was filtered off and washed with ethanol. The crude product was dissolved in $10 \mathrm{ml}$ toluene and precipitated again by adding $400 \mathrm{ml}$ of ethanol. From the ${ }^{1} \mathrm{H}-\mathrm{NMR}$ (Hitachi, R-1900, 90 MHz) measurements, we confirmed that the product was H-GNC. The core size of the HGNC determined by transmission electron microscopy (TEM) (JEOL, JEM-2000FX) was 1.8 $\pm 0.4 \mathrm{~nm}$.

MUA and $\mathrm{FcC}_{6} \mathrm{SH}$ were introduced onto the $\mathrm{H}-\mathrm{GNC}$ surface by the place-exchange method [9]. As an example, we describe the procedure for the introduction of $\mathrm{FcC}_{6} \mathrm{SH}$. The HGNC $(0.6 \mathrm{~g})$ was first dispersed in $100 \mathrm{ml}$ of dichloromethane, and then $\mathrm{FcC}_{6} \mathrm{SH}(0.25 \mathrm{~g}, 8.3$ mmol) was added. The solution was stirred for $3 \mathrm{~h}$ at room temperature. After evaporating off 
the solvent, the precipitate was washed twice with ethanol and acetone. The crude product was dissolved in $10 \mathrm{ml}$ of dichloromethane and precipitated again by adding $400 \mathrm{ml}$ of ethanol. The $1.8 \pm 0.4 \mathrm{~nm}$ core diameter of the GNCs determined by TEM did not change after the reagent exchange reactions. As the molar ratios of the alkylthiols on the GNC surfaces varied each time, they were determined each time by ${ }^{1} \mathrm{H}-\mathrm{NMR}$. The molar ratios of $\mathrm{C}_{6} \mathrm{SH}: \mathrm{FcC}_{6} \mathrm{SH}:$ MUA on the MHF-GNC surface and of $\mathrm{C}_{6} \mathrm{SH}:$ MUA on the MH-GNC surface were determined by ${ }^{1} \mathrm{H}-\mathrm{NMR}$ as $47: 19: 34$ and $26: 74$, respectively.

GNCs Multilayers with the following three different arrangements as described in Scheme 1 were formed on the carboxylate-terminated (MUA) SAM-modified gold substrates based on the carboxylate/PAH/carboxylate electrostatic interaction. 1. Multilayer containing only MHF-GNC/PAH (Scheme 1 (a): Au(111)/MUA/(PAH/MHF-GNC) $)$ was prepared on a Au surface by repeating the two-dip and rinse cycle as reported before [27]. 2. Multilayer of MH-GNC and MHF-GNC with only one layer of MHF-GNC existed in the layer closest to the gold electrode, i.e., the first layer, and MH-GNC in the other layers (Scheme 1(b): $\left.\mathrm{Au}(111) / \mathrm{MUA} / \mathrm{PAH} / \mathrm{MHF}-\mathrm{GNC} /(\mathrm{PAH} / \mathrm{MH}-\mathrm{GNC})_{\mathrm{n}}\right)$. 3. Multilayer of MH-GNC and MHFGNC with only one layer of MHF-GNC existed only in the outermost layer and MH-GNC in the other layers (Scheme 1(c): $\left.\mathrm{Au}(111) / \mathrm{MUA} /(\mathrm{PAH} / \mathrm{MH}-\mathrm{GNC})_{\mathrm{n}} / \mathrm{PAH} / \mathrm{MHF}-\mathrm{GNC}\right)$. The substrate pre-coated with MUA SAM was dipped in a $2 \mathrm{wt} \%$ PAH aqueous solution $(\mathrm{pH}=6)$ containing $1 \mathrm{M} \mathrm{NaCl}$ for 30 min followed by a Milli-Q water rinse and then dipped in a 0.75 wt $\%$ GNC ethanol solution for 30 min followed by a rinse with ethanol. This two-dip and rinse cycle was repeated to construct the multilayers.

Cyclic voltammograms (CVs) were recorded with an automatic polarization system (Hokuto Denko, HSV-100). A potentiostat/galvanostat (Hokuto Denko, HA-151) and a function generator (Hokuto Denko, HB-111) were used for the EQCM measurements, which were carried out in a three-compartment cell. The resonant frequency of the quartz crystal electrode, which was oscillated by a homemade oscillation circuit, was simultaneously 
monitored with the electrode potential and current by a frequency counter (Hewlett-Packard, HP53131A) controlled by a personal computer (NEC, PC9821cb2) through a GPIB interface. The frequency stability of the EQCM system was better than $0.1 \mathrm{~Hz}$ for a sampling gate time of $0.1 \mathrm{~s}$. Pt wire and $\mathrm{Ag} / \mathrm{AgCl}$ were used as the counter and reference electrodes, respectively. The measurements were carried out in a $0.1 \mathrm{M} \mathrm{HClO}_{4}$ aqueous solution, which was deaerated by passing ultrapure $\mathrm{N}_{2}$ gas through it for more than 30 min before each measurement.

The XPS measurements were performed on a Rigaku XPS-7000 spectrometer using an $\operatorname{MgK} \alpha \mathrm{X}$-ray source $(1253.6 \mathrm{eV})$. The take-off angle between the sample surface and the analyzer was fixed at $90^{\circ}$ in all cases. The narrow-scan spectra of the Fe2p region were taken with a resolution of $0.1 \mathrm{eV}$ and the signals of 64 scans were averaged.

Cross-sectional transmission electron microscopy of a GNC multilayer was carried out using a JEOL electron microscope JEM-2010F with $200 \mathrm{KV}$ acceleration voltage. A multilayer with ten MHF-GNC/PAH deposition cycles was prepared on a silicon wafer with a pre-evaporated $\mathrm{Au}$ film of $150 \mathrm{~nm}$. After the multilayer formation, the multilayer was further covered with the evaporated $\mathrm{Au}$ and epoxy resin to protect the multilayer for the preparation of a sliced sample. 


\section{RESULTS AND DISCUSSION}

(1) Structure and electrochemical characteristics of the MHF-GNC/PAH multilayers

Figure 1 shows the cross-sectional micrograph of a multilayer with ten MHF-GNC deposition cycles. The substrate (evaporated $\mathrm{Au}$ film), GNC/PAH multilayers, and the protecting layers, i.e., the evaporated Au and epoxy resin, were clearly visible in Fig. 1 (a). From this image, the total thickness of the GNC multilayer with ten GNC/PAH cycles was estimated to be about $58 \mathrm{~nm}$, which is in good agreement with the previous reported value of $51 \mathrm{~nm}$ determined by ellipsometry for the same system [27]. This and the higher resolution image (Fig. 1(b)) revealed that the GNCs were dispersed within the polymeric matrix rather uniformly and the well-defined layered structure as illustrated in Scheme 1 was not observed, suggesting a homogenous mixture of the GNCs and PAH in the LBL film rather than the expected vertically ordered structure with sharp interfaces between the two components. This is reasonable because the layered LBL assembly might be obscured in the case of globular nanoparticles alternatively assembled with flexible linear polymeric chains due to the interpenetration between the adjacent layers and high interlayer roughness or by embedding of the GNCs on the polymeric chains. The spherical nanoparticles were separated from each other by the organic shells and the mean size of the immobilized GNC core was about $1.8 \pm$ $0.3 \mathrm{~nm}$, which was almost the same as that of the GNC in solution, revealing the absence of aggregation or reshapening of the linked GNC particles to those of larger core size in the LBL multilayers. The average separation distance of the immobilized GNCs was estimated by measuring the edge-to-edge distances of the particles in the cluster regions in Fig.1 (b). The result yields an almost uniform spacing close to the cluster core size. Similar features are expected for the multilayers with the ferrocene group either existed at the innermost and or outermost layers, since both types of GNCs used for the fabrication of LBL multilayers with PAH spacing layers have same core size and similar activity.

Figure 2 (a) shows the CVs of the electrode modified with MHF-GNC/PAH multilayers 
of 1-10 deposition cycles of (Scheme 1 (a)) measured in a $0.1 \mathrm{M} \mathrm{HClO}_{4}$ aqueous solution at a scan rate of $100 \mathrm{mV} \mathrm{s}^{-1}$. The oxidation and reduction peaks due to the redox of $\mathrm{Fc} / \mathrm{Fc}^{+}$were observed around $+360 \mathrm{mV}$ (vs. $\mathrm{Ag} / \mathrm{AgCl}$ ) in all the cases. The full width at half-maximum (fwhm) of MHF-GNC multilayer modified electrode was constant to be around $90 \mathrm{mV}$ regardless of the GNC deposition cycles, suggesting a simple Langmuir type redox process of the ferrocene moieties. The peak-to-peak separation was less than $60 \mathrm{mV}$ in all the cases, suggesting a facile charge transfer within the MHF-GNC multilayers. The charge of the redox peak linearly increased with the MHF-GNC deposition cycles as summarized in Fig. 2 (b). Note that all charge values in this study are slightly larger than that those previously reported [27] because the surface coverage of $\mathrm{FcC}_{6} \mathrm{SH}$ in the present system was higher than that of the previous case.

(2) Structure and electrochemical characteristics of the multilayers consisted of both MHFGNC and MH-GNC with two different sequences

It is impossible to clarify the GNC arrangement in the multilayers with the ferrocene group either existed at the innermost and or outermost layers by the cross-sectional transmission electron microscopy since both types of GNCs used for the LBL multilayer formation with PAH spacing layers had same core size. However, the X-ray photoelectron spectra of the multilayer assembly in the $\mathrm{Fe} 2 \mathrm{p}$ region can provide additional structural information concerning the position of MHF-GNC in the multilayer. Figure 3 shows the Xray photoelectron spectra in the Fe2p region of the two types of multilayer assemblies: (a) $\mathrm{Au}(111) / \mathrm{MUA} / \mathrm{PAH} / \mathrm{MHF}-\mathrm{GNC} /(\mathrm{PAH} / \mathrm{MH}-\mathrm{GNC})_{\mathrm{n}}$ for $\mathrm{n}=1,3$ and 5, and (b) Au(111)/MUA/ $(\mathrm{PAH} / \mathrm{MH}-\mathrm{GNC})_{\mathrm{n}} / \mathrm{PAH} / \mathrm{MHF}-\mathrm{GNC}$ for $\mathrm{n}=1,3,5$ and 9. No peak was observed even at $\mathrm{n}=3$ in the former (Fig. 3(a)), while the peak intensity did not change with $\mathrm{n}$ in the latter (Fig. 3(b)), confirming that the ferrocene group definitely existed at the innermost and outermost layers in the former and the latter systems, respectively, as suggested in Schemes 1(b) and 1(c).

Figures 4 (a) and 4 (b) shows the CVs of the former $(n=1,3,5,9)$ and the latter $(n=1,3$, 
7, 9) multilayer modified electrodes measured in a $0.1 \mathrm{M} \mathrm{HClO}_{4}$ aqueous solution at a scan rate of $100 \mathrm{mV} \mathrm{s}^{-1}$. For a comparison, the $\mathrm{CV}$ of the $\mathrm{Au}(111) / \mathrm{MUA} / \mathrm{PAH} / \mathrm{MHF}-\mathrm{GNC}$, which is equivalent to the assembly of $n=0$ of the both sequences, is also shown in the both figures. For all the cases, the oxidation and reduction peaks due to the redox of $\mathrm{Fc} / \mathrm{Fc}^{+}$were observed around $+360 \mathrm{mV}$ (vs. $\mathrm{Ag} / \mathrm{AgCl}$ ). The peak width and peak-to-peak separation of the redox $\mathrm{Fc}$ group increased significantly with the introduction of one PAH/MH-GNC layer then slightly with a further increase of the PAH/MH-GNC layer. The peak-to-peak separation increased from $35 \mathrm{mV}(\mathrm{n}=0)$, to $75 \mathrm{mV}(\mathrm{n}=1), 100 \mathrm{mV}(\mathrm{n}=5)$, and $125 \mathrm{mV}(\mathrm{n}=9)$ for the former, and to $165 \mathrm{mV}(\mathrm{n}=1), 175 \mathrm{mV}(\mathrm{n}=7)$, and $185 \mathrm{mV}(\mathrm{n}=9)$ for the latter. However, the charge in these peaks did not depend on $\mathrm{n}$ and were $23-24 \mu \mathrm{C} \mathrm{cm}{ }^{-2}$, which was in good agreement with that of the $\mathrm{Au}(111) / \mathrm{MUA} / \mathrm{PAH} / \mathrm{MHF}-\mathrm{GNC}$ consisting of only one MHF-GNC/PAH deposition cycle, i.e., the case of $n=0$ of the both sequences, as summarized in Fig. 4 (c). All these charge values are slightly larger than that previously reported [27] due to the similar reason as mentioned above. As the charge did not depend on the number of MH-GNC/PAH layers, one could conclude that all the Fc groups in the multilayer on the gold electrode were electrochemically active despite the presence of the PAH/MH-GNC multilayer between the electrolyte solution and the ferrocene moiety in the former and between the gold electrode and ferrocene moiety in the latter.

(3) Charge transfer through the GNC multilayers

Since the redox process of the surface-attached ferrocene group is known to be represented by [28]:

$$
-\mathrm{Fc}+\mathrm{X}_{\mathrm{s}}^{-}-\mathrm{Fc}^{+} \mathrm{X}^{-}+\mathrm{e}^{-}+\mathrm{solv}
$$

where $-\mathrm{Fc}$ and $-\mathrm{Fc}^{+}$are the neutral and oxidized states of the ferrocene group, respectively, $\mathrm{X}_{\mathrm{s}}$ - is the solvated anion in solution, $-\mathrm{Fc}^{+} \mathrm{X}^{-}$is an ion pair between the ferricenium cation and the anion, and solv. is a solvent molecule, electron and anion should be transferred between the ferrocene moiety and the electrode, and between the electrolyte solution and the ferrocene 
moiety, respectively, upon the redox of the ferrocene group. Thus, the results shown in Figs. 2 and 4 strongly suggest that perchlorate anion, which forms an ion pair with $\mathrm{Fc}^{+}$, is transferred between the electrolyte solution and the ferrocene group at the innermost layer through the 1 $9 \mathrm{PAH} / \mathrm{MH}-\mathrm{GNC}$ layers at the Au/MUA/PAH/MHF-GNC/(PAH/MH-GNC) $)_{\mathrm{n}}(\mathrm{n}=1,3,5,9)$ electrodes and electron is transferred between the ferrocene group at the outermost layer and the gold electrode through the 1 - 9 PAH/MH-GNC layers at the Au/MUA/(PAH/MHGNC)/PAH/MHF-GNC ( $\mathrm{n}=1,3,7,9)$ electrodes.

Figure 5 shows the potential dependencies of current and EQCM at the $\mathrm{Au} / \mathrm{MUA} / \mathrm{PAH} / \mathrm{MHF}-\mathrm{GNC} /(\mathrm{PAH} / \mathrm{MH}-\mathrm{GNC})_{9}$ electrode obtained simultaneously in $0.1 \mathrm{M}$ $\mathrm{HClO}_{4}$ aqueous solution at a scan rate of $10 \mathrm{mV} \mathrm{s}^{-1}$. Note the current density of Fig. 5 was smaller than the results shown in Fig. 4 because the sweep rate was 10 times smaller in the latter. Significant frequency decrease and increase, i.e., weight increase and decrease, were observed when the current due to the oxidation and reduction of the ferrocene moiety, respectively, flowed. A linear relation was observed between the mass change calculated from the frequency change by using Saubrey's equation and the charge in the region of the ferrocene oxidation $(250-550 \mathrm{mV})$ with the slope of $216 \mathrm{~g} / \mathrm{mol}$-electron. Similar EQCM response was observed for the Au/MUA/PAH/MHF-GNC/(PAH/MH-GNC) $)_{3}$ and $\mathrm{Au} / \mathrm{MUA} / \mathrm{PAH} / \mathrm{MHF}-\mathrm{GNC} /(\mathrm{PAH} / \mathrm{MH}-\mathrm{GNC})_{6}$ electrodes. The slopes for the mass change charge relations, i.e., mass per electron: mpe, for the former and the latter were 233 and 177, respectively. Furthermore, the essentially the EQCM response was obtained at the $\mathrm{Au} / \mathrm{MUA} /(\mathrm{PAH} / \mathrm{MH}-\mathrm{GNC})_{9} / \mathrm{PAH} / \mathrm{MHF}-\mathrm{GNC}$. These results clearly show that the EQCM response was not affected by the number of the PAH/MH-GNC overlayers, confirming perchlorate ions are transferred between the electrolyte solution and the ferrocene group at the innermost layer through the PAH/MH-GNC. The mpe values obtained for $\mathrm{Au} / \mathrm{MUA} / \mathrm{PAH} / \mathrm{MHF}-\mathrm{GNC} /(\mathrm{PAH} / \mathrm{MH}-\mathrm{GNC})_{\mathrm{n}}(\mathrm{n}=3,6,9)$ are in good agreement with the value observed at the gold electrode modified with the PAH/MHF-GNC multilayers (245 
$\mathrm{g} / \mathrm{mol}$-electron) and is much larger than the molecular weight of perchlorate ion $(99.5 \mathrm{~g} / \mathrm{mol})$, suggesting that each perchlorate ion moves into and is released from the modified layer accompanied by 5 - 7 water molecules upon the oxidation and reduction of the ferrocene moiety, respectively.

At least two possible mechanisms can be considered for the electron transfer between the ferrocene group on the MHF-GNC surface and the gold electrode: one is the direct tunneling between the ferrocene group on the MHF-GNC surface and the gold electrode and the other is the transfer through the core of the MH-GNCs. The distance between the ferrocene group in the outermost layer and the gold electrode in the GNC multilayers with ten deposition cycles was more than $50 \mathrm{~nm}$ as evidenced by both cross-sectional transmission electron microscopy and ellipsometry and is far too long for electron to tunnel. Thus, the electron should be transferred not by direct tunneling between the ferrocene group and the gold electrode but through the GNCs by hopping.

In comparison with those of the multilayers with only MHF-GNC, the peak width and the peak-to-peak separation increased significantly with the introduction of PAH/MH-GNC layers in the multilayers with MHF-GNC either as the layer closest to the gold electrode, i.e., the first layer, or as the outermost layer with $\mathrm{MH}-\mathrm{GHC}$ in the other layers. One possible reason for these peak broadening and the peak-to-peak separation is the slow kinetics for the charge transfer, including the electron transfer between the Au electrode and the Fc moiety and the transport of the perchlorate ion between the electrolyte and the Fc moiety, through these layers. The facile electron transfer through these multilayers was, however, demonstrated by measuring the scan rate dependence of the electrochemical characteristics. Figure 6 and Table 1 shows the CVs and electrochemical parameters, respectively, of the $\mathrm{Au} / \mathrm{MUA} / \mathrm{PAH} / \mathrm{MHF}-\mathrm{GNC} /(\mathrm{PAH} / \mathrm{MH}-\mathrm{GNC})_{3}$ modified electrode. The peak potential shifted only slightly and the peak current increased almost linearly with the scan rate up to $200 \mathrm{mV} \mathrm{s}^{-}$ ${ }^{1}$, and the corresponding charge was almost independent of the scan rate. Thus, the charge 
transfer is indeed facile. Another possible contribution for the peak broadening and the enlarged peak separation might result from the non-uniform packing arrangement of the MHF-GNC in these multilayers with different sequences, although we could not provide a clear evidence for the non-uniform packing of the MHF-GNC. The packing arrangement of the redox moiety attached GNC in these multilayers should play an important role in the transport of both electron and $\mathrm{ClO}_{4}^{-}$within the multilayers and some changes of the microstrcture of these films might take place during the redox process of the Fc groups. Above results implied that although perchlorate anion and electrons can be transferred through the $\mathrm{MH}-\mathrm{GNC} / \mathrm{PAH}$ layers so that all the ferrocene groups are electrochemically active, their transports within the $\mathrm{MH}-\mathrm{GNC} / \mathrm{PAH}$ layers are slower than those in the PAH/MHF-GNC layers, suggesting the promotion of their transports by the ferrocene/ferricenium groups in the latter system.

\section{Conclusion}

Two types of GNCs, one covered by SAMs of MUA, $\mathrm{C}_{6} \mathrm{SH}$, and $\mathrm{FcC}_{6} \mathrm{SH}$, MHF-GNC, and the other with MUA and $\mathrm{C}_{6} \mathrm{SH}, \mathrm{MH}-\mathrm{GNC}$, were used for the construction of ten GNC layers of two different sequences on an MUA-modified $\mathrm{Au}(111)$ surface based on the carboxylate/polycation (PAH)/carboxylate electrostatic interaction. MHF-GNC was placed either as the layer closest to the gold electrode, i.e., the first layer, or as the outermost layer with MH-GHC in the other layers. A quasi-reversible redox peak with a constant charge corresponding to the redox of the ferrocene moiety of the MHF-GNC monolayer was observed at both electrodes, showing that electrons and perchlorate ions are transferred through the MH-GNC/PAH multilayers as shown in Scheme 2 for (a) $\mathrm{Au}(111) / \mathrm{MUA} / \mathrm{PAH} / \mathrm{MHF}-\mathrm{GNC} /(\mathrm{PAH} / \mathrm{MH}-\mathrm{GNC})_{\mathrm{n}}$, and (b) $\mathrm{Au}(111) / \mathrm{MUA} /(\mathrm{PAH} / \mathrm{MH}-$ GNC) $)_{\mathrm{n}} / \mathrm{PAH} / \mathrm{MHF}-\mathrm{GNC}$ electrodes. 


\section{Acknowledgement}

WS acknowledges the Japan Society for the Promotion of Science for a Postdoctoral Fellowship for Foreign researchers. Part of this work was supported by a Grants- in-Aid for Scientific Research from the Ministry of Education, Culture, Sports, Science, and Technology, Japan (Nos. 1322203 and 13874085). We would like to thank Prof. Shimazu and Dr. Sugawara for their help with the XPS and TEM measurements, respectively. 


\section{References}

1. M. Brust, M. Walker, D. Bethell, D. J. Schiffrin, R. Whyman, J. Chem. Soc., Chem. Commun. (1994) 801.

2. R. G. Freeman, K. C. Graber, K. J. Allison, R. M. Bright, J. A. Davis, A. P. Guthrie, M. B. Hommer, M. A. Jackson, P. C. Smith, D. G. Walter, M. J. Natan, Science 267 (1995) 9466.

3. C. A. Mirkin, R. L. Letsinger, R. C. Mucic, J. J. Storhoff, Nature 382 (1996) 607.

4. R. A. Reynolds III, C. A. Mirkin, R. L. Letsinger, Pure Appl. Chem. 72 (2000) 229.

5. T. G. Schaaff, M. N. Shafigullin, J. T. Khoury, I. Vezmar, R. L. Whetten, W. G. Cullen, P. N. First, J. Phys. Chem. B 101 (1997) 7885.

6. D. I. Gittins, D. Bethell, D. J. Shiffrin, R. J. Nichols, Nature 408 (2000) 67.

7. S. J. Green, J. J. Stokes, M. J. Hostetler, J. J. Peitron, R. W. Murray, J. Phys. Chem. B 101 (1997) 1997.

8. S. Chen, R. W. Murray, S. W. Feldberg, J. Phys. Chem. B 102 (1998) 9898.

9. M. J. Hostetler, A. C. Templeton, R. W. Murray, Langmuir 15 (1999) 3782.

10. A. C. Templeton, W. P. Zamborini, F. P.; W. P. Wuelfing, R. W. Murray, Langmuir 16 (2000) 6682.

11. F. P. Zamborini, J. F. Hicks, R. W. Murray, J. Am. Chem. Soc. 122 (2000) 4514.

12. (a) J. F. Hicks, F. P. Zamborini, A. J. Osisek, R. W. Murray, J. Am. Chem. Soc. 123 (2001) 7048. (b) W. Peter Wuelfing, R. W. Murray, J. Phys. Chem. B 106 (2002) 3139. (c) J. F. Hicks, Y. Seok-Shon, R. W. Murray, Langmuir 18 (2002) 2288. (d) W. Peter Wuelfing, S. J. Green, J. J. Pietron, D. E. Cliffel, R. W. Murray, J. Am. Chem. Soc. 122 (2000) 11465.

13. T. Horikoshi, M. Itoh, M. Kurihara, K. Kubo, N. Nishihara, J. Electroanal. Chem. 473 (1999) 113.

14. Y. Men, K. Kubo, M. Kurihara, H. Nishihara, Phys. Chem. Chem. Phys. 3 (2001) 3427.

15. A. Ulman, An Introduction to Ultrathin Organic Films from Langmuir-Blodgett to SelfAssembly, Academic Press: San Diego, CA. 1991. 
16. H. O. Finklea, Electroanalytical Chemistry, ed. A. J. Bard and I. Rubinstein, Marcel Dekker, New York, 1996, vol.19.

17. (a) T. Kondo, T. Ito, S. Nomura, K. Uosaki, Thin Solid Films 284/285 (1996) 652. (b) T. Kondo, M. Yanagida, S. Nomura, T. Ito, K. Uosaki, J. Electroanal. Chem. 438 (1997) 121.

18. (a) T. Kondo, K. Iso-o, T. Kanai, K. Uosaki, Z. Phys. Chem. 212 (1999) 23. (b) T. Kondo, M. Yanagida, X.-Q. Zhang, K. Uosaki, Chem. Lett. 2000, 964.

19. (a) K. Uosaki, T. Kondo, X.-Q. Zhang, M. Yanagida, J. Am. Chem. Soc. 119 (1997) 8367.

(b) T. Kondo, S. Horiuchi, I. Yagi, S. Ye, K. Uosaki, J. Am. Chem. Soc. 121 (1999) 391.

20. M. Yanagida, T. Kanai, X.-Q. Zhang, T. Kondo, K. Uosaki, Bull. Chem. Soc. Jpn. 71 (1998) 2558. (b) T. Kondo, T. Kanai, K. Uosaki, Langmuir 17 (2001) 6317.

21. (a) M. Yamada, H. Nishihara, Chem. Commun., 2002, 2578. (b) M. Yamada, A. Kuzume, M. Kurihara, K. Kubo, H. Nishihara, Chem. Commun., 2001, 2476.

22. (a) G. Decher, Science, 277 (1997) 1232. (b) G. Decher and J. Schmitt, Prog. Colloid Polym. Sci. 89 (1992) 160. (c) P. Bertrand, A. Jonas, A. laschewsky and R. legras, Macromol. Rapid. Commun. 21 (2000) 319.

23. (a) A. Mamedov, J. Ostrander, F. Aliev and N. A. Kotov, Langmuir, 16 (2000) 3941. (b) A. Ikeda, T. hatano, S. Shinkai, T. Akiyama and S. Yamada, J. Am. Chem. Soc. 123 (2001) 4855.

24. (a) M. Sastry, M. Rao and K. N. Ganesh, Acc. Chem. Res. 35 (2002) 847. (b) T. Sennerfors, G. Bogdanovic and F. Tiberg, Langmuir, 18 (2002) 6410. (c) Z. Tang, Y. Wang and N. A. Kotov, Langmuir, 18 (2002) 7035.

25. J. W. Ostrander, A. A. Mamedov and N. A. Kotov, J. Am. Chem. Soc. 123 (2001) 1101.

26. T. Kondo, M. Okamura, K. Uosaki, Chem. Lett. 2001, 930.

27. K. Uosaki, T. Kondo, M. Okamura, W. B. Song, Faraday Discuss. 121 (2002) 373.

28. K. Uosaki, Y. Sato, H. Kita, Langmuir 7 (1991) 1510; (b) K. Uosaki, Y. Sato, H. Kita, Electrochim. Acta. 36 (1991) 1799. 
29. J. Clavilier, J. Electroanal. Chem. 107 (1980) 205; (b) J. Clavilier, J. Electroanal. Chem. 107 (1980) 211.

30. K. Uosaki, S. Ye, T. Kondo, J. Phys. Chem., 99 (1996) 1411. 
Table 1 Electrochemical parameters of Au/MUA/PAH/MHF-GNC/(PAH/MH-GNC) modified electrode measured in $0.1 \mathrm{M} \mathrm{HClO}_{4}$ aqueous solution at different potential scan rate. 


\begin{tabular}{ccccccc}
\hline Scan rate $/ \mathrm{mV} \mathrm{s}^{-1}$ & 5 & 10 & 20 & 50 & 100 & 200 \\
\hline $\mathrm{E}_{\mathrm{pa}} / \mathrm{V}$ & 0.37 & 0.37 & 0.37 & 0.38 & 0.39 & 0.41 \\
\hline $\mathrm{E}_{\mathrm{pc}} / \mathrm{V}$ & 0.35 & 0.34 & 0.34 & 0.33 & 0.31 & 0.29 \\
\hline$\Delta \mathrm{E}_{\mathrm{p}} / \mathrm{mV}$ & 20 & 30 & 30 & 50 & 80 & 120 \\
\hline $\mathrm{Ip} / \mu \mathrm{A} \mathrm{cm} \mathrm{cm}^{-2}$ & 1.2 & 2.0 & 3.5 & 6.7 & 12.5 & 22.1 \\
\hline Charge / $/ \mu \mathrm{Cm}^{-2}$ & 36.2 & 32.4 & 25.2 & 24.1 & 23.5 & 23.2 \\
\hline
\end{tabular}

Table 1 W.Song et.al. 


\section{Figure Captions}

Scheme 1. Schematic view of the idealized layer structure of (a) Au(111)/MUA/(PAH/ MHF-

GNC $)_{\mathrm{n}}, \quad$ (b) $\quad \mathrm{Au}(111) / \mathrm{MUA} / \mathrm{PAH} / \mathrm{MHF}-\mathrm{GNC} /(\mathrm{PAH} / \mathrm{MH}-\mathrm{GNC})_{\mathrm{n}}, \quad$ and

$\mathrm{Au}(111) / \mathrm{MUA} /(\mathrm{PAH} / \mathrm{MH}-\mathrm{GNC})_{\mathrm{n}} / \mathrm{PAH} / \mathrm{MHF}-\mathrm{GNC}$ electrodes.

Scheme 2. Schematically idealized model for the charge transfer during the redox of the ferrocene moiety at (a) $\mathrm{Au}(111) / \mathrm{MUA} / \mathrm{PAH} / \mathrm{MHF}-\mathrm{GNC} / \mathrm{PAH} / \mathrm{MH}-\mathrm{GNC})_{\mathrm{n}}$, and (b) $\mathrm{Au}(111) / \mathrm{MUA} /(\mathrm{PAH} / \mathrm{MH}-\mathrm{GNC})_{\mathrm{n}} / \mathrm{PAH} / \mathrm{MHF}-\mathrm{GNC}$ electrodes.

Figure 1 Cross-sectional transmission electron micrographs of a (a) MHF-GNC multilayer films with ten deposition cycles on an evaporated Au film on the silicon wafer, and (b) higher resolution image of the GNC/PAH multilayer region.

Figure 2 Cyclic voltammograms of $\mathrm{Au}(111) / \mathrm{MUA} /(\mathrm{PAH} / \mathrm{MHF}-\mathrm{GNC})_{\mathrm{n}}$ for $\mathrm{n}=1-10$ measured in $0.1 \mathrm{M} \mathrm{HClO}_{4}$ aqueous solution at a scan rate of $100 \mathrm{mV} \mathrm{s}^{-1}$ (a) and relationship between the electronic charge of Fc redox peak and the number of MHF-GNC/PAH deposition cycles (b).

Figure 3 X-ray photoelectron spectra of (a) Au(111)/MUA/PAH/MHF-GNC/(PAH/ MHGNC) $)_{\mathrm{n}}$ for $\mathrm{n}=1,3$ and 5 and (b) $\mathrm{Au}(111) / \mathrm{MUA} /(\mathrm{PAH} / \mathrm{MH}-\mathrm{GNC})_{\mathrm{n}} / \mathrm{PAH} / \mathrm{MHF}-\mathrm{GNC}$ for $\mathrm{n}=1$, 3,5 and 9.

Figure 4 Cyclic voltammograms of (a) Au(111)/MUA/PAH/MHF-GNC/(PAH/MH- GNC) for $\mathrm{n}=1,3,5$ and 9 and (b) $\mathrm{Au}(111) / \mathrm{MUA} /(\mathrm{PAH} / \mathrm{MH}-\mathrm{GNC}) \mathrm{n} / \mathrm{PAH} / \mathrm{MHF}-\mathrm{GNC}$ for $\mathrm{n}=1,3,7$ and 9 measured in $0.1 \mathrm{M} \mathrm{HClO}_{4}$ aqueous solution at a scan rate of $100 \mathrm{mV} \mathrm{s}^{-1}$. For a comparison, $\mathrm{CV}$ of $\mathrm{Au}(111) / \mathrm{MUA} / \mathrm{PAH} / \mathrm{MHF}-\mathrm{GNC}$ is presented in both figures as $\mathrm{Au}(111) / \mathrm{MUA} / \mathrm{PAH} / \mathrm{MHF}-\mathrm{GNC}$ is equivalent to the assembly of $\mathrm{n}=0$ of both systems. (c) Relationship between the electronic charge of Fc redox peak and the number of MH-GNC deposition cycles for the two assemblies.

Figure 5 EQCM response of the $\mathrm{Au}(111) / \mathrm{MUA} / \mathrm{PAH} / \mathrm{MHF}-\mathrm{GNC} /(\mathrm{PAH} / \mathrm{MH}-\mathrm{GNC})_{9}$ electrode measured in $0.1 \mathrm{M} \mathrm{HClO}_{4}$ aqueous solution at a scan rate of $10 \mathrm{mV} \mathrm{s}^{-1}$. 
Figure 6 Cyclic voltammograms of (a) Au(111)/MUA/PAH/MHF-GNC/(PAH/MH- GNC) measured in $0.1 \mathrm{M} \mathrm{HClO}_{4}$ aqueous solution at different scan rates. 


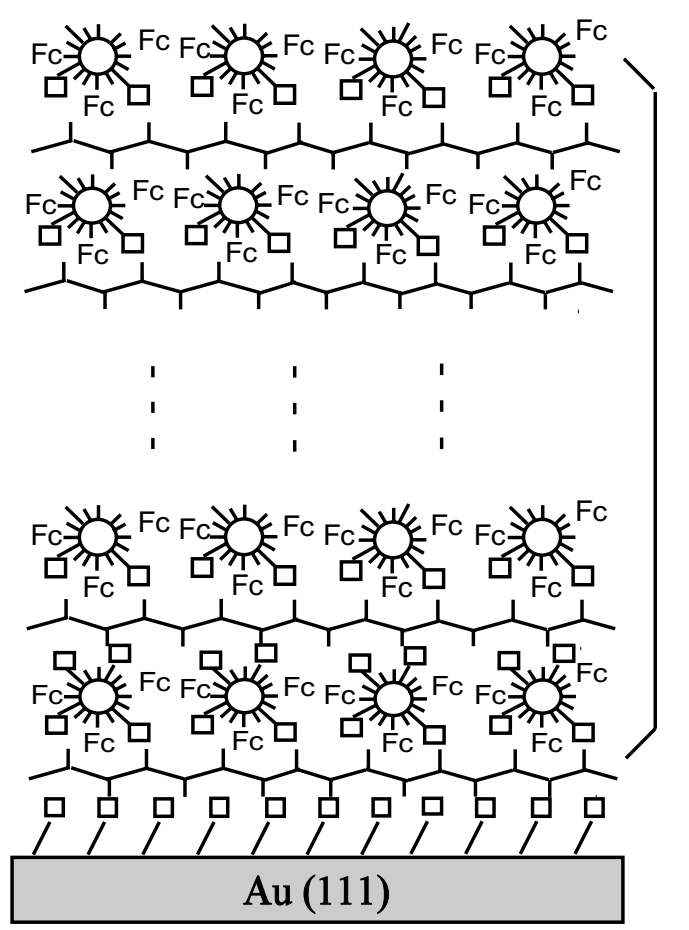

(b)
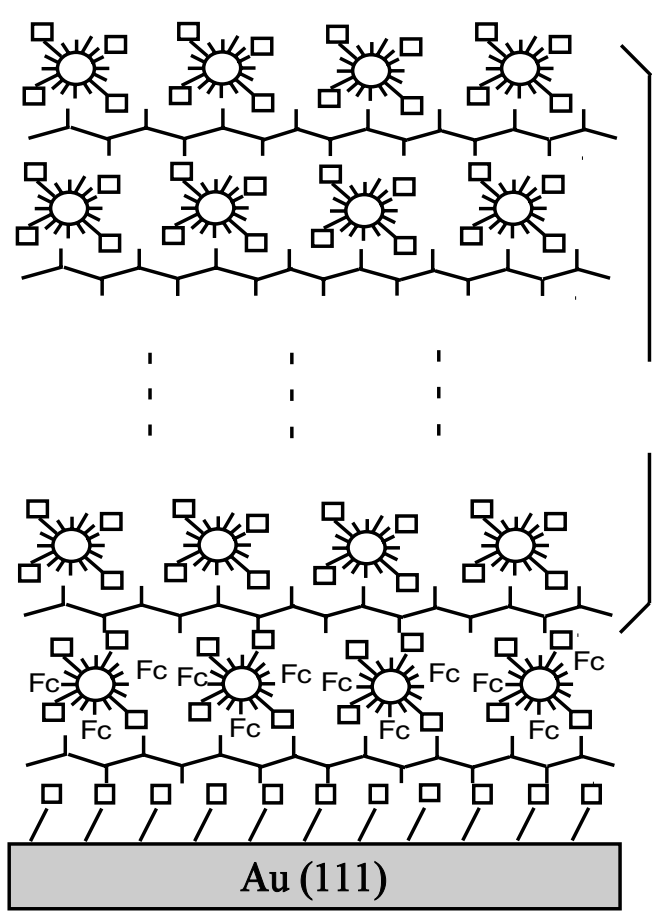

$\mathrm{n}=1-10$

\begin{tabular}{|c|c|}
\hline$\square$ & Carboxylate group \\
\hline & PAH \\
\hline & MHF-GNC \\
\hline & MH-GNC \\
\hline
\end{tabular}

\section{(c)}

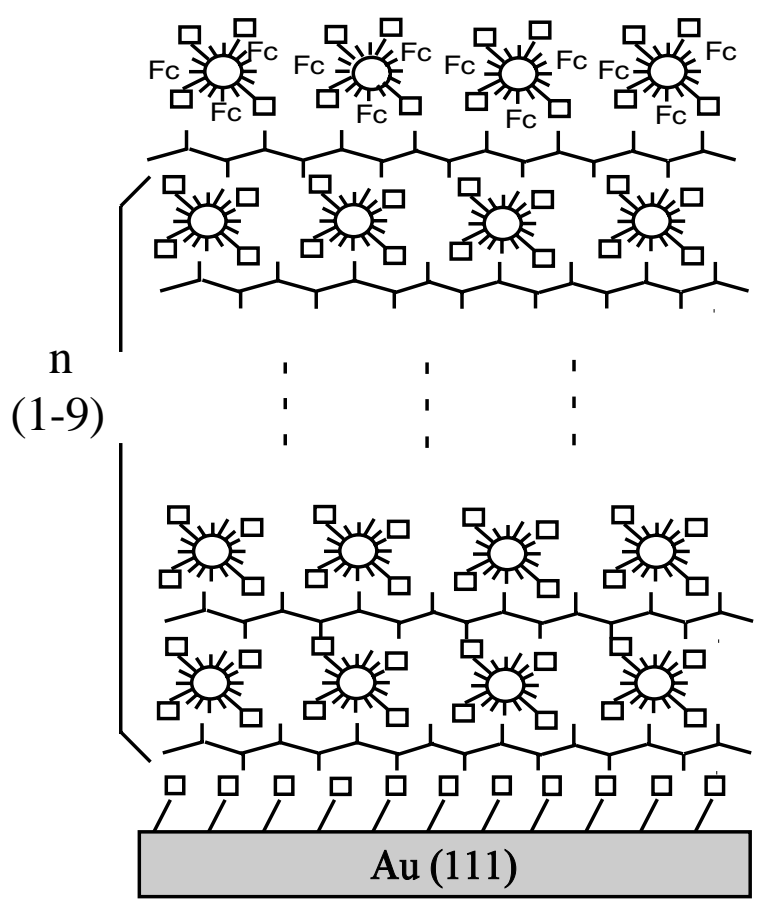




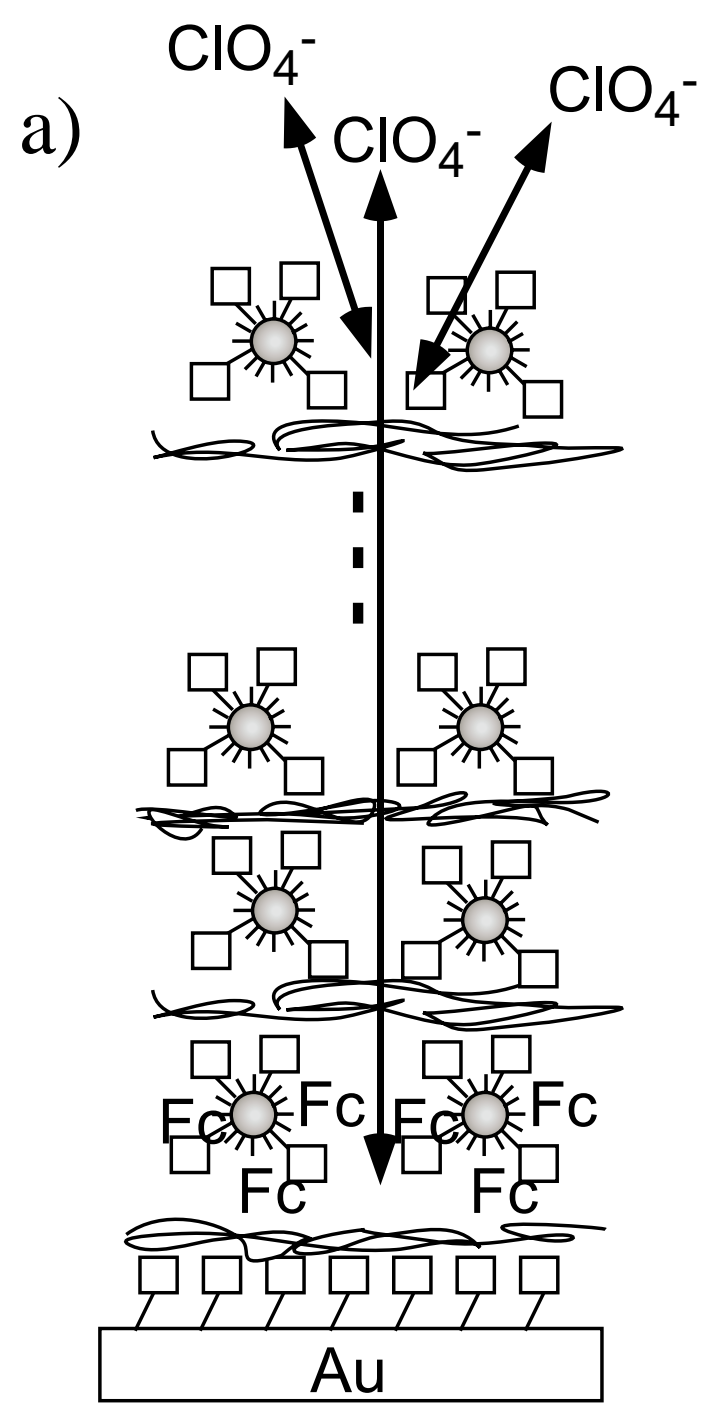

b)

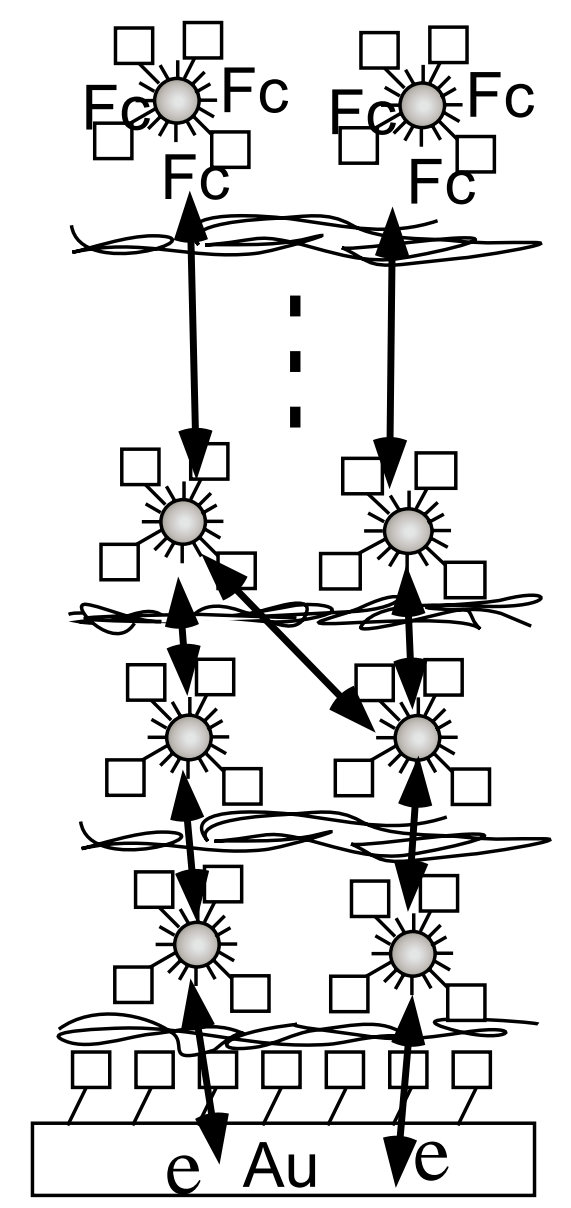

: Au nanocluster

Fc : ferrocene group

$\square$ :carboxylate group

Scheme 2 W. Song et al. 
(a)

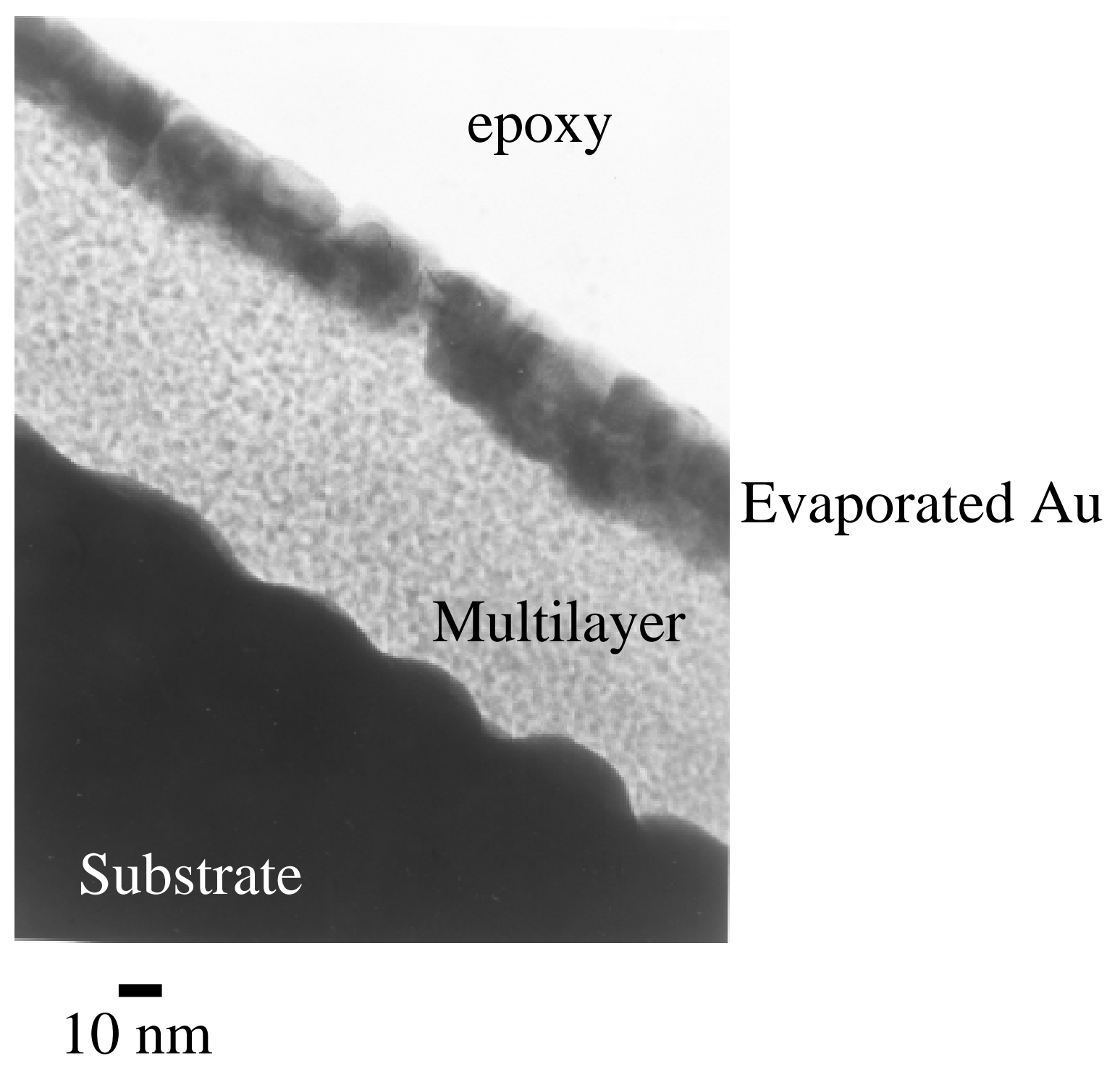

(b)

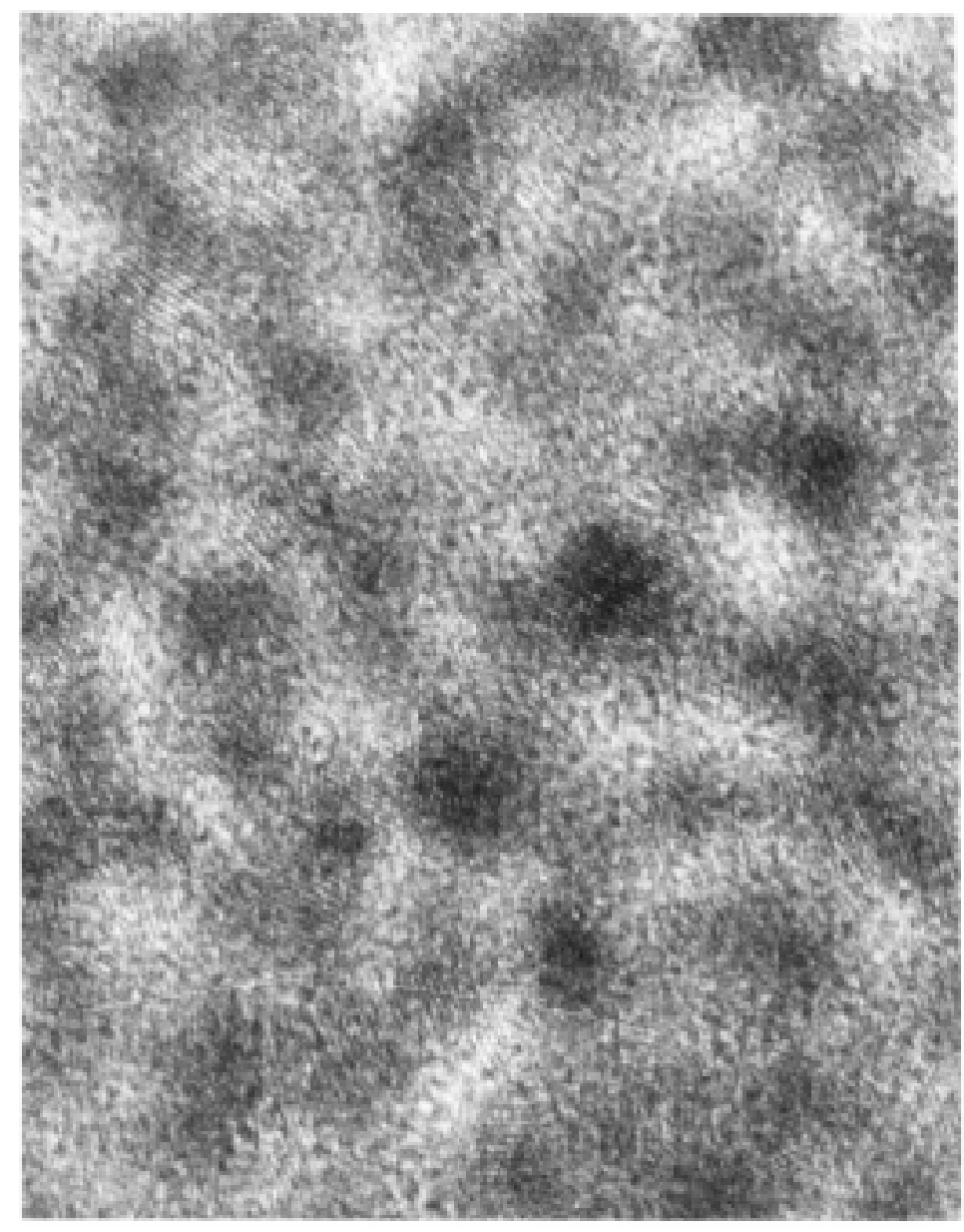

$5 \mathrm{~nm}$

Fig. $1 \mathrm{~W}$. Song et al. 


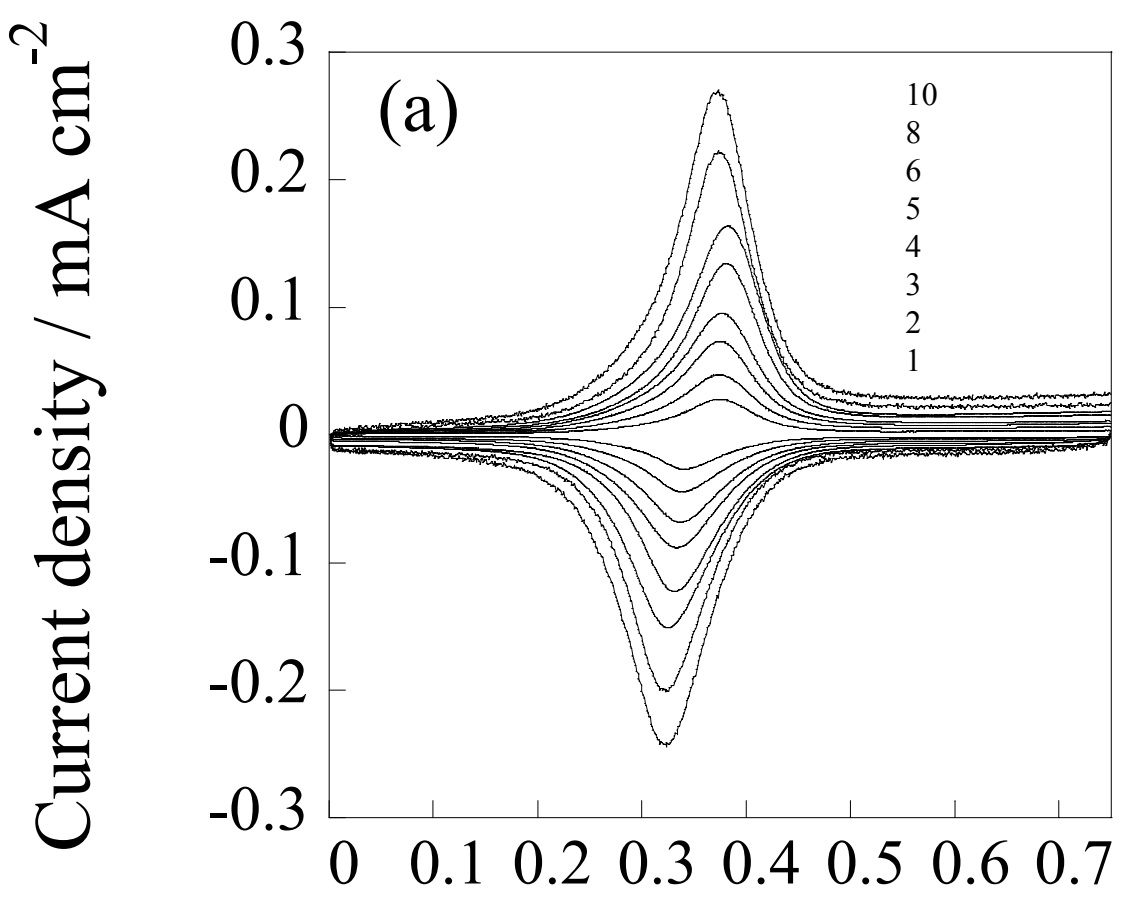

Potential / V vs. $\mathrm{Ag} / \mathrm{AgCl}$

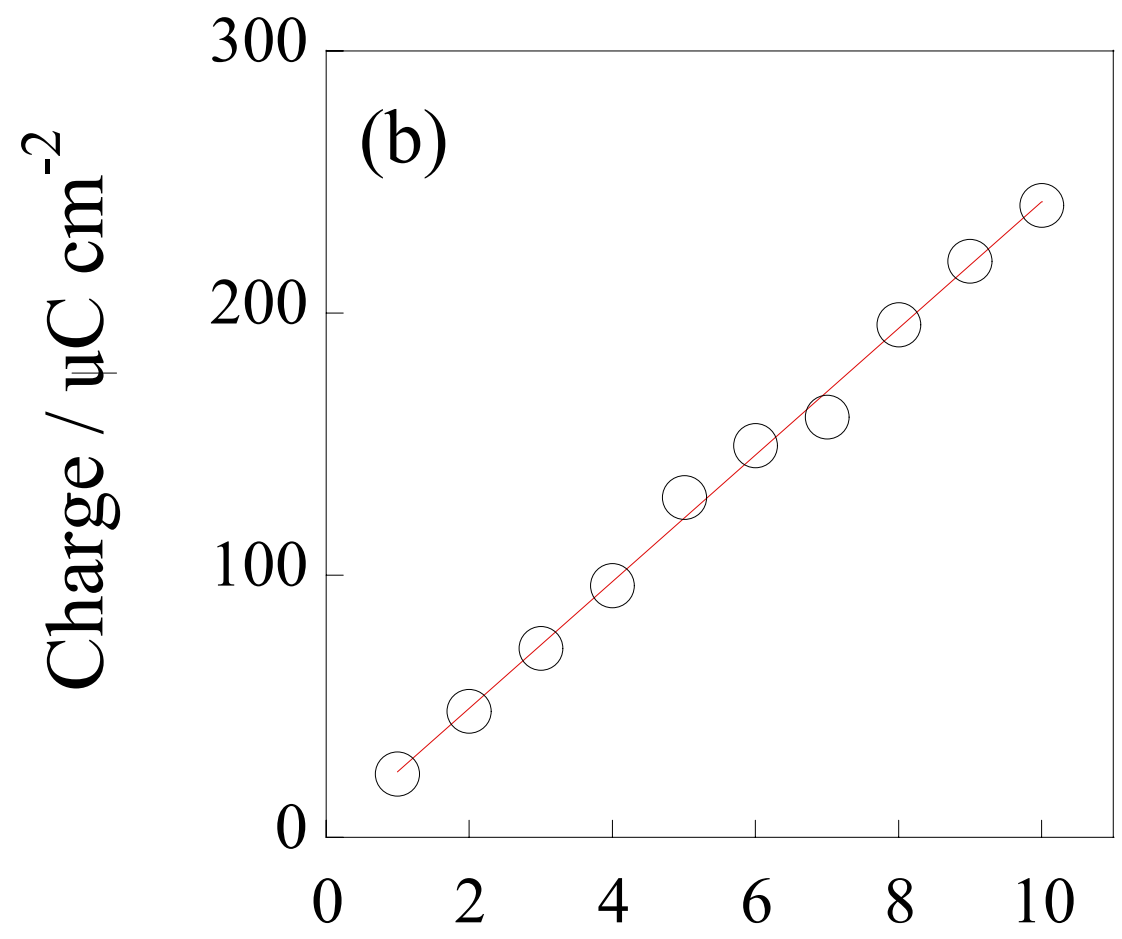

Number of MHF-GNC layers

Fig.2 W. Song et al. 

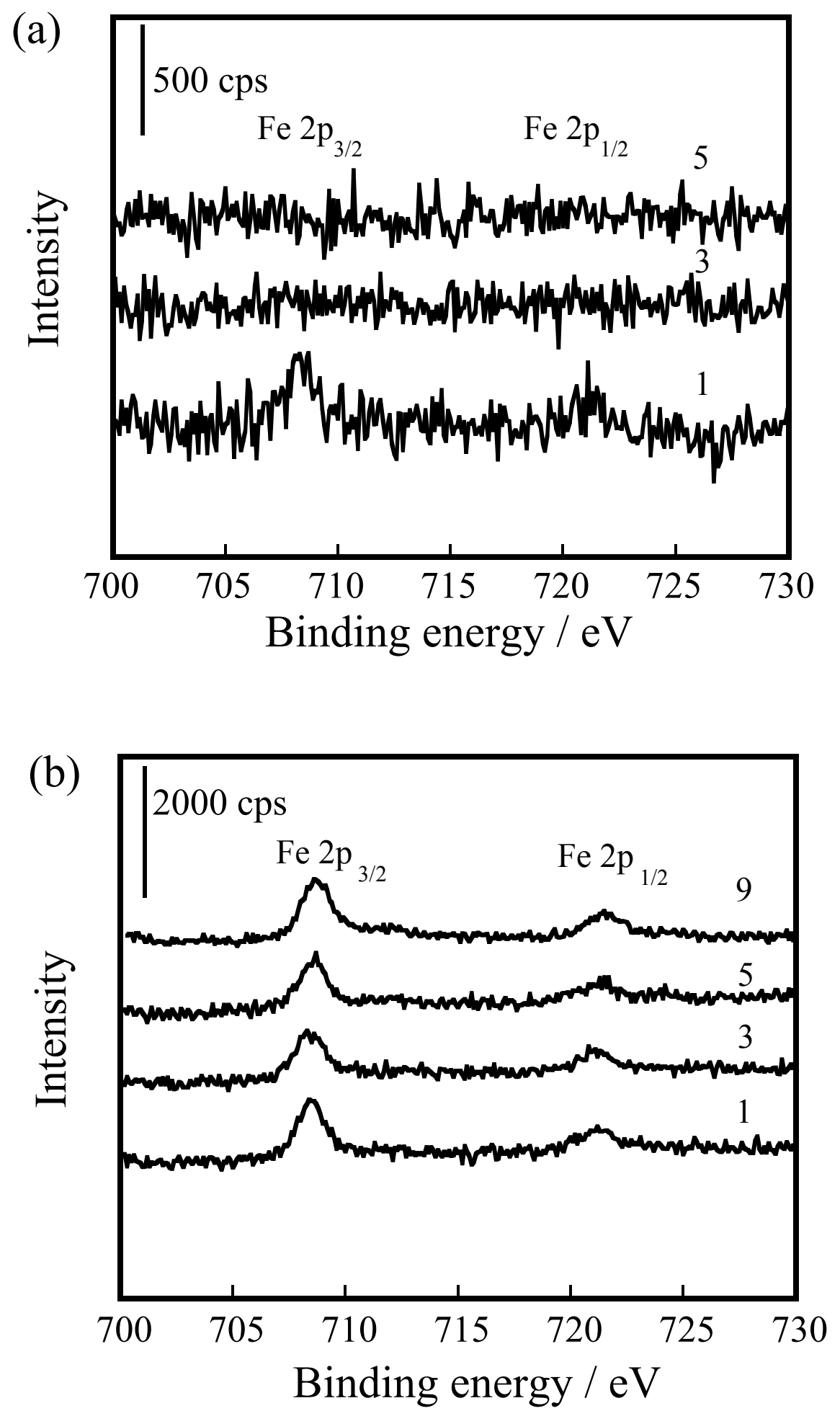

Fig. 3 W. Song et.al 


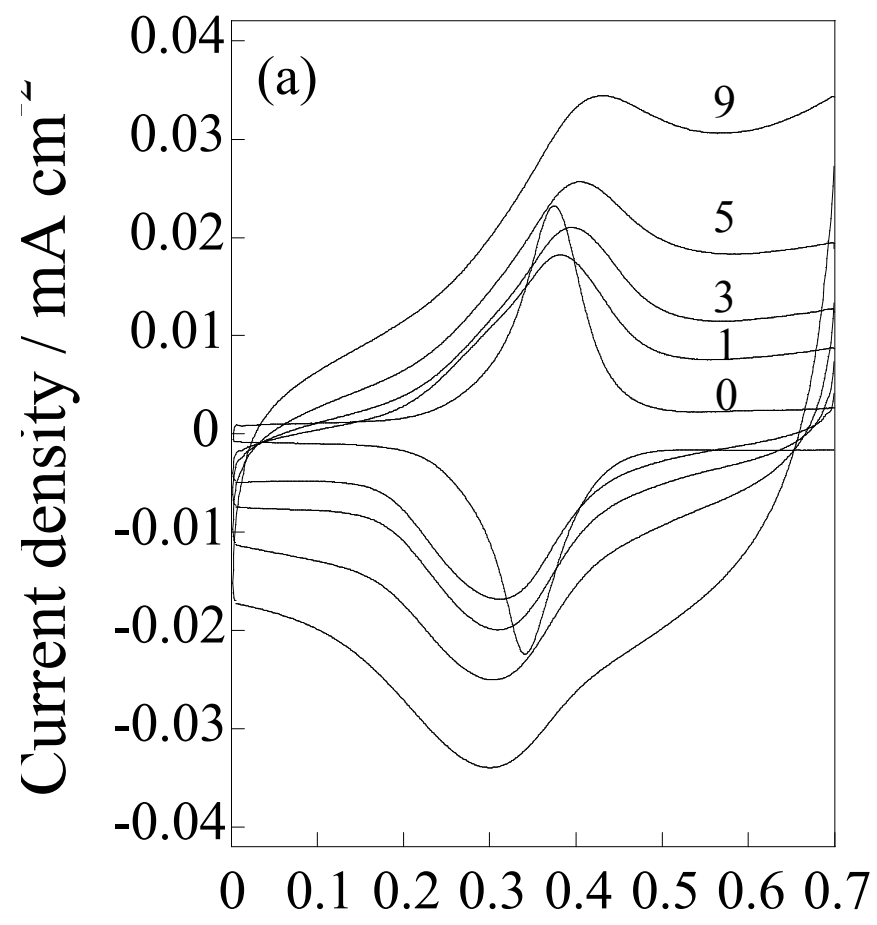

Potential / V vs. $\mathrm{Ag} / \mathrm{AgCl}$

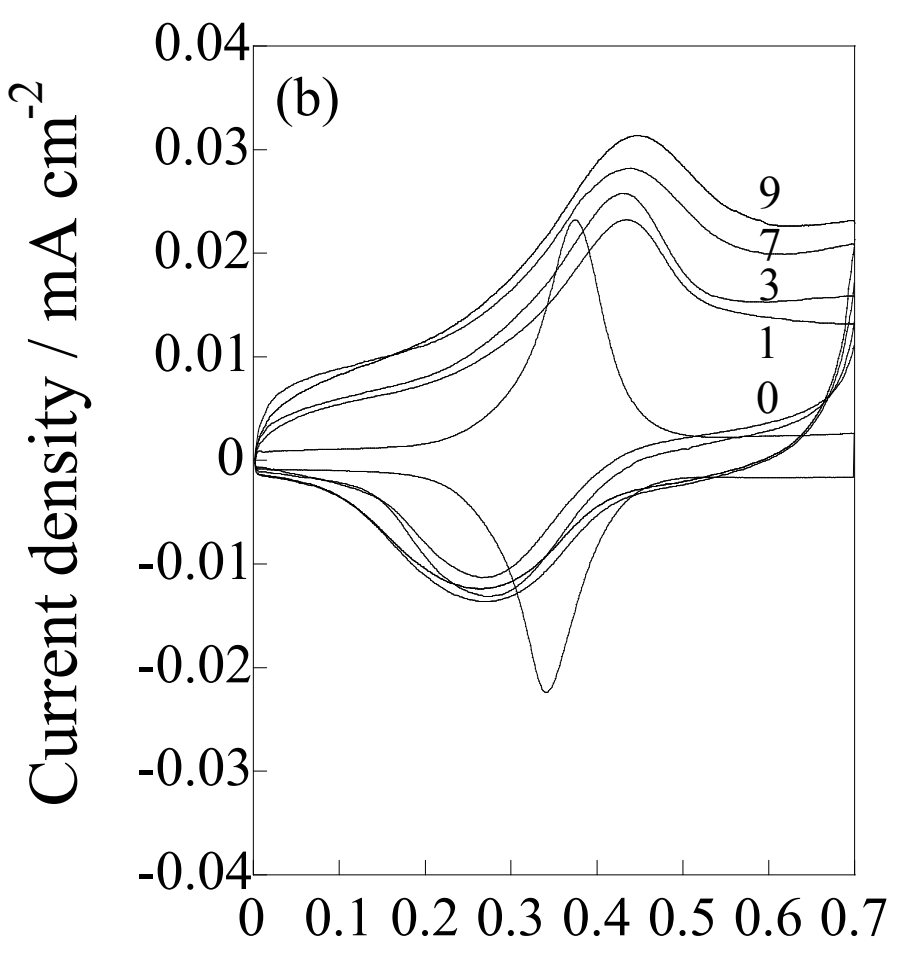

Potential / V vs. $\mathrm{Ag} / \mathrm{AgCl}$

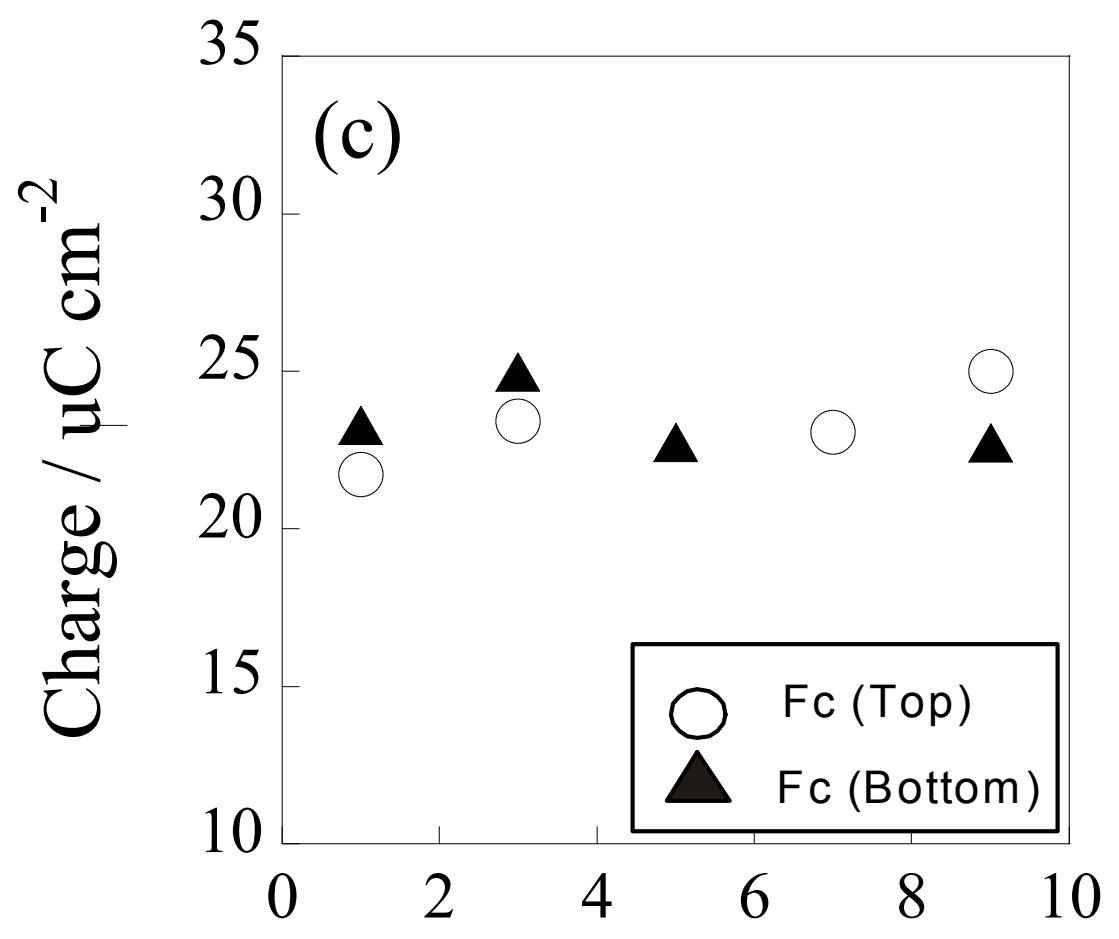

Number of MH-GNC layers

Fig.4 W. Song et al. 


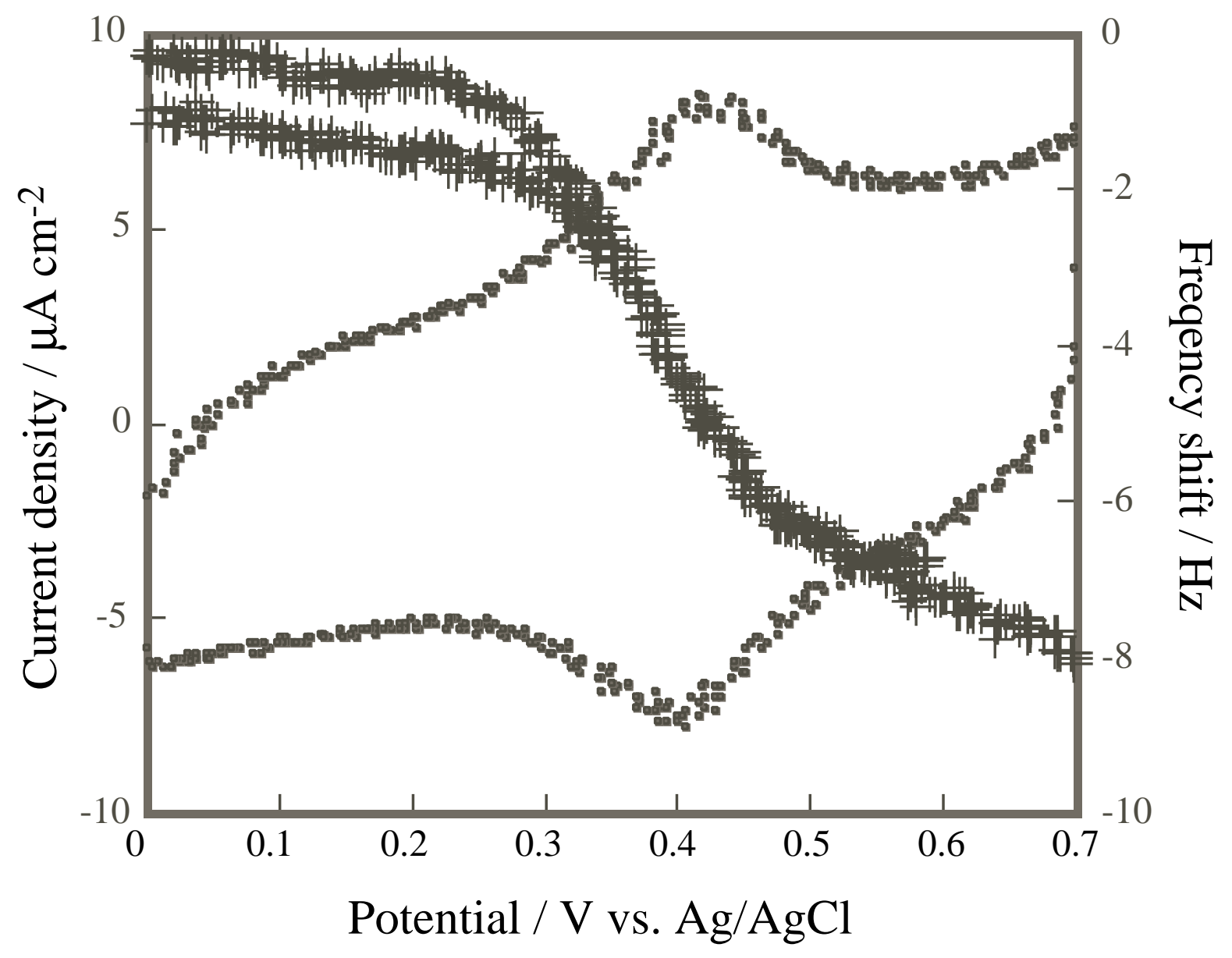

Fig. 5 W. Song et al. 


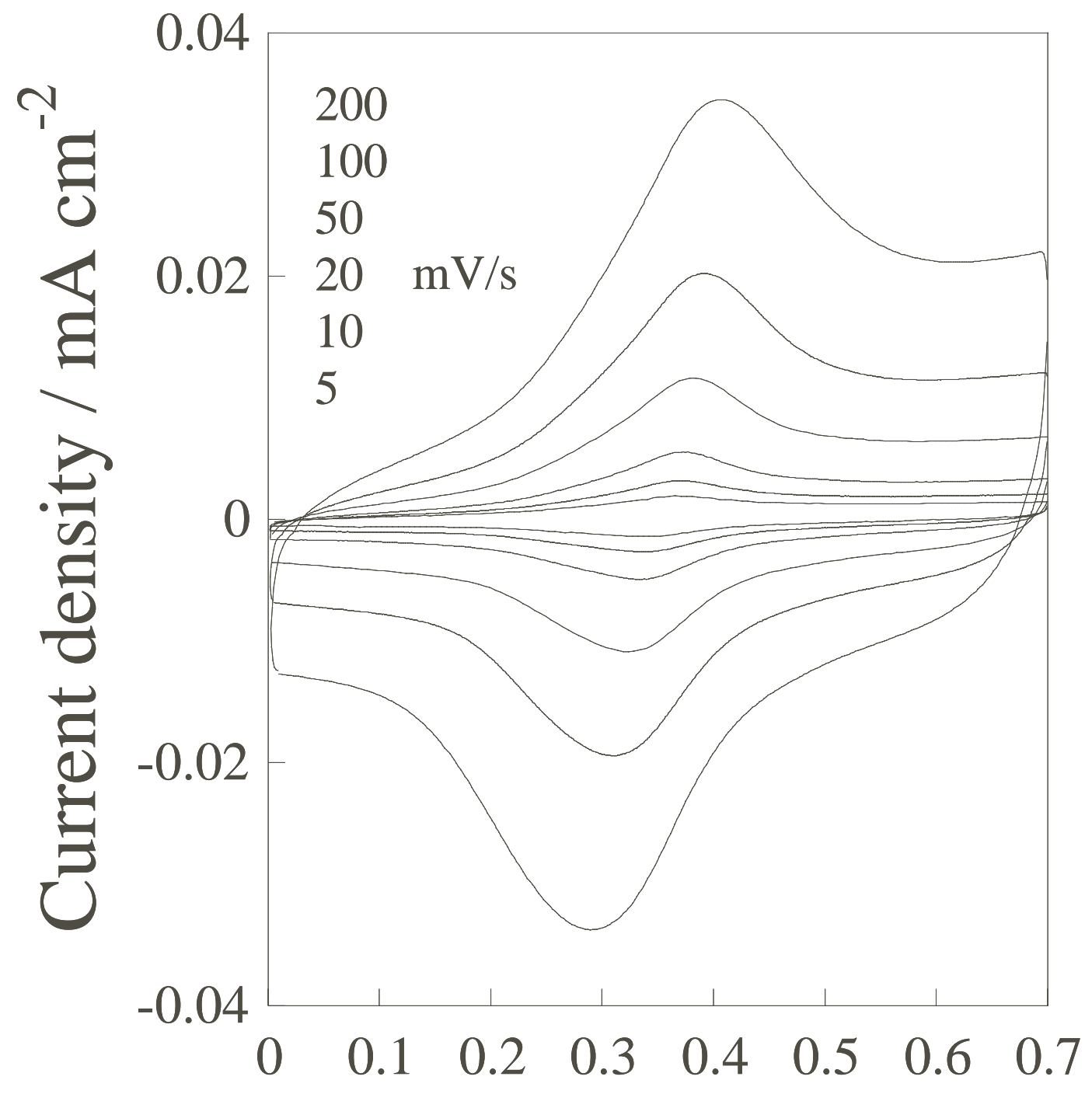

Potential / V vs. $\mathrm{Ag} / \mathrm{AgCl}$

Fig. 6 W. Song et. al. 